\title{
Labial Mucosa
}

National Cancer Institute

\section{Source}

National Cancer Institute. Labial Mucosa. NCI Thesaurus. Code C67370.

The inner lining of the lips. 